\title{
Inteligencia artificial aplicada a programas informativos de radio. Estudio de caso de segmentación automática de noticias en RNE
}

\section{Artificial intelligence applied to radio news: a case study of automatic segmentation of news items at $R N E$}

\author{
Virginia Bazán-Gil; Carmen Pérez-Cernuda; Noemí Marroyo-Núñez; \\ Paloma Sampedro-Canet; David De-Ignacio-Ledesma
}

Cómo citar este artículo:

Bazán-Gil, Virginia; Pérez-Cernuda, Carmen; Marroyo-Núñez, Noemí; Sampedro-Canet, Paloma; De-IgnacioLedesma, David (2021). "Inteligencia artificial aplicada a programas informativos de radio. Estudio de caso de segmentación automática de noticias en RNE". Profesional de la información, v. 30, n. 3, e300320.

https://doi.org/10.3145/epi.2021.may.20

Artículo recibido el 22-12-2020

Aceptación definitiva: 15-02-2021

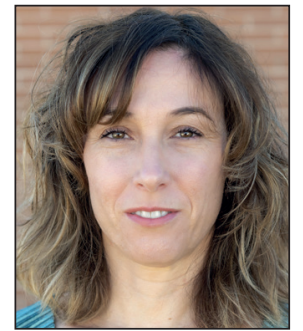

Virginia Bazán-Gil $\bowtie$

https://orcid.org/0000-0003-4920-2212

Radiotelevisión Española

Fondo Documental RTVE

Avda. Radio Televisión, 4

28223 Pozuelo de Alarcón (Madrid), España

virginia.bazan@rtve.es

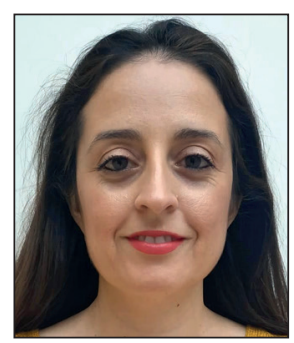

Noemí Marroyo-Núñez

https://orcid.org/0000-0001-9588-2584

Radiotelevisión Española

Innovación y Estrategia tecnológica

Avda. Radio Televisión, 4

28223 Pozuelo de Alarcón (Madrid), España

noemi.marroyo@rtve.es

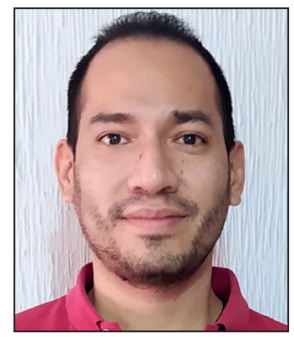

David De-Ignacio-Ledesma

https://orcid.org/0000-0002-8146-2447

Soprasteria

Avda. de Manoteras, 48

28050 Madrid, España

david.deignacioledesma@soprasteria.com

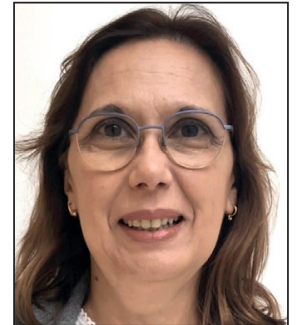

Carmen Pérez-Cernuda

https://orcid.org/0000-0001-5128-5905

Radiotelevisión Española

Innovación y Estrategia tecnológica

Avda. Radio Televisión, 4

28223 Pozuelo de Alarcón (Madrid), España

carmen.perez.cernuda@rtve.es

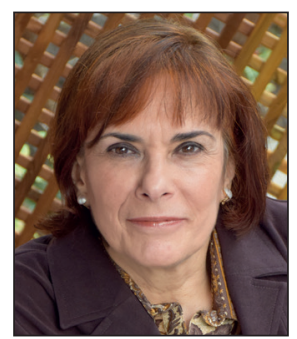

Paloma Sampedro-Canet

https://orcid.org/0000-0003-2208-2249

Corporación Radio Televisión Española

Avda. Radio Televisión, 4

28223 Pozuelo de Alarcón (Madrid), España

paloma.sampedro@rtve.es

\section{Resumen}

Se presentan los resultados del proyecto para la segmentación en noticias de los informativos de Radio Nacional de España (RNE) llevado a cabo por el Área de Innovación Tecnológica de Radio Televisión Española (RTVE) en colaboración con la Dirección de Medios de RNE. El objetivo de este proyecto es aplicar la inteligencia artificial para el cortado automático de las noticias que componen un informativo radiofónico, para su posterior difusión en la web de RTVE y en medios de comunicación social. El proyecto se planificó en dos fases: una primera de configuración y ajuste del sistema, y una segunda de prestación del servicio propiamente dicho. Los criterios de calidad mínimos exigibles se definieron previamente, tanto para la transcripción automática del habla a texto, para la que se estableció una tasa de error por palabra máxima (WER) del 10\%, como para la segmentación de noticias, para la que se definió una tasa de precisión 
superior al 85\%. El rendimiento del sistema tanto en la transcripción como en la segmentación se considera suficiente, si bien se espera alcanzar un mayor grado de precisión en el cortado de noticias en los próximos meses. Los resultados ponen de manifiesto que, a pesar de ser tecnologías bastante maduras, son necesarios procesos de ajuste y aprendizaje con la intervención humana.

\title{
Palabras clave
}

RTVE; RNE; Inteligencia artificial; Tecnologías del lenguaje; Trascripción de voz a texto; Programas de radio; Archivos audiovisuales; Archivos de radio; Documentación audiovisual; Documentación radiofónica; Diarización; Segmentación de noticias; WER; Word error rate; Precisión; Control de calidad.

\begin{abstract}
The results of a project on news segmentation at Radio Nacional de España (RNE) carried out by the RTVE Technological Innovation and Media Management areas is presented. The aim of this project is to apply artificial intelligence to automatically transcribe and cut the news items that make up a radio news program. The main goals of this project are to increase the accessibility of the content and to allow its reusability on various platforms and social media. The project was planned in two phases, covering system configuration and service delivery. The minimum quality criteria required were defined in advance, both for automatic voice transcription and for news segmentation. For the speech-to-text process, the highest word error rate (WER) allowed was 10\%, while the precision rate for the news segmentation was $85 \%$. System performance in both transcription and segmentation was considered to be sufficient, although a higher degree of accuracy in news cutting is expected in the coming months. The results show that, despite using these quite mature technologies, adjustment and learning processes and human intervention are still necessary.
\end{abstract}

\section{Keywords}

RTVE; RNE; Artificial intelligence; Language technologies; Voice-to-text transcription; Radio news; Audiovisual archives; Radio archives; Audiovisual documentation; Radio documentation; Diarization; News segmentation; WER; Word error rate; Precision; Quality control.

\section{Introducción}

Los medios de comunicación se hallan inmersos en una transformación sin precedentes motivada por el agotamiento del que ha sido, hasta el momento, su modelo de negocio e impulsada por la crisis del coronavirus. Los contenidos se crean, se consumen y desechan a una velocidad hasta ahora impensable, las audiencias están fragmentadas y el mercado de la información y el entretenimiento presenta una oferta desbordante. Cada vez son más necesarios elementos innovadores que desde la propia narrativa, el medio o la tecnología permitan crear y distribuir contenidos orientados a las personas, individualmente, y no a audiencias generales (Corral, 2020). Los medios de comunicación buscan en la inteligencia artificial soluciones para producir, distribuir, posicionar mejor sus contenidos y atraer a nuevas audiencias, sin perder de vista las múltiples aplicaciones que estas tecnologías tienen para mejorar la accesibilidad y poner en valor los contenidos de archivo. En este contexto, las empresas de radio y televisión exploran las posibilidades de la inteligencia artificial en:

- La identificación, seguimiento y predicción de temas de interés público en redes sociales y otras fuentes de información para generar nuevos contenidos que permitan alimentar esos debates, atraer y fidelizar nuevas audiencias (ReTV, 2020; Nixon, 2020; Rozalén-Serrano; Aranda-Jiménez, 2020).

- El análisis de grandes conjuntos de datos para comprender de forma profunda los acontecimientos y el desarrollo de estudios de género en radio y televisión (Doukhan, 2019; BBC, 2020).

- La creación de contenidos personalizados o de interés local (Associated Press, 2020; WashPostPR, 2017; Jones; Jones, 2019; Túñez-López; Toural-Bran; Valdiviezo-Abad, 2019; Yle, 2018; Molumby; Whitwell, 2019; Parmentier, 2020) y la generación automática de noticias (Vállez; Codina, 2018; Túñez-López; Toural-Bran; Cacheiro-Requeijo, 2018; Herrero-Diz; Varona-Aramburu, 2018).

- La detección de noticias falsas (fake news) (Graves, 2018; Grothaus, 2019; Fraunhofer-Gesellschaft, 2019) y la lucha contra la desinformación.

- La mejora de la accesibilidad de los contenidos publicados, mediante el subtitulado automático y la traducción a otros idiomas (Saarikoski, 2020; Gálvez, 2020).

- La puesta en valor de los contenidos de archivo a través de la generación automática de metadatos a partir del análisis de la imagen y del sonido (Lempinen; Kokko; Matusiak, 2020; Van-Rijsselbergen, 2020; Bazán-Gil, 2018; Rath; Veerwaijen; Forster, 2020; Opoku-Boateng; Asano, 2020; León-Carpio; López-De-Quintana, 2020).

En los medios de comunicación surgen a diario proyectos que ponen de manifiesto cómo un uso adecuado de la inteligencia artificial y el aprendizaje automático puede mejorar tanto las herramientas como los flujos de trabajo actuales. El proyecto Journalism Al de la London School of Economics and Political Science (LSE, 2020) recoge los más recientes casos de uso en ética, diversidad y moderación de contenidos, personalización y distribución de noticias, periodismo de investigación, verificación de noticias y lucha contra la desinformación. Igualmente describe estrategias en inteligencia artificial en las redacciones, educación y formación e innovación que se han llevado a cabo en los últimos años en perió- 
dicos digitales como The Financial Times, The New York Times; en empresas de radio y televisión como $B B C$, Yle o Sky News; o en agencias de noticias como Reuters o News Agency. En esta misma línea, la de presentación de estudios de caso, pero en el contexto de las empresas públicas de radio y televisión en Europa se sitúa el informe News Report 2019 "The next newsroom: Unlocking the power of Al for public service journalism" publicado por la European Broadcasting Union (EBU, 2019). Este informe, analiza las oportunidades y riesgos de la inteligencia artificial al servicio del periodismo y, trata de identificar buenas prácticas a través de las entrevistas realizadas a 75 expertos del ámbito de la tecnología, el periodismo y la investigación.

En el sector audiovisual, el Research for CULT Committee del Parlamento Europeo publicó en septiembre de 2020 "The use of artificial intelligence in the audiovisual sector" (Rehm, 2020), un análisis en profundidad de este tema. Se basa en una encuesta de 10 preguntas distribuida en 73 organizaciones europeas y recoge una amplia implantación de la inteligencia artificial para la indexación automática y la mejora de la accesibilidad de los contenidos audiovisuales. En este sentido se reconocen como tecnologías relevantes:

- los sistemas de reconocimiento automático del habla (ASR),

- los sistemas de conversión de texto a habla (TTS),

- el procesamiento del lenguaje natural (PLN),

- el reconocimiento de entidades nombradas (NER) o

- los servicios de traducción automática (MT).

RTVE no ha permanecido ajena a esta transformación y, en los últimos años, desde la Dirección de Estrategia Tecnológica e Innovación Digital se han impulsado iniciativas y proyectos para la incorporación de la inteligencia artificial en los procesos de captación y difusión de noticias.

En concreto, en RTVE se han focalizado las actuaciones en actividades relacionadas con la producción de informativos en todas sus fases (Prensa RTVE, 2018; Sanjinés, 2020a; 2020b), desde las más tempranas de detección de hechos noticiables, hasta las últimas de presentación de las noticias, tanto por los medios tradicionales (TDT, satélite), como por los nuevos sistemas no lineales (Web, redes sociales, etc.) y la preservación digital de estos contenidos en el archivo. Ya en 2017, en colaboración con la Universidad Carlos III de Madrid, se empezó a trabajar en el diseño de un servicio de monitorización y alerta multinivel de contenidos en redes sociales para la detección temprana de noticias, un proyecto que dio lugar a la aplicación Social Media Radar (SMR) (Rozalén-Serrano et al., 2020).

En julio de ese mismo año se creó la Cátedra de RTVE (2017) en la Universidad de Zaragoza con el fin de impulsar las tecnologías para la automatización de los procesos de metadatado en los archivos audiovisuales, una de cuyas actividades principales es el lanzamiento de retos tecnológicos a la comunidad científica que permitan evaluar el estado del arte de las tecnologías del habla, el procesamiento del lenguaje natural (PLN) y la visión artificial aplicadas al análisis de contenidos audiovisuales. Como resultado de este esfuerzo, RTVE ha puesto a disposición de los investigadores dos conjuntos de datos denominados RTVE Database 2018 (Lleida-Solano et al., 2018) y RTVE Database 2020 (Lleida-Solano et al., 2020), en los que se han basado los retos tecnológicos Iberspeech-RTVE Challenge 2018 y Albayzín-RTVE 2020 (Lleida-Solano, 2020), organizados por la Red Temática en Tecnologías del Habla en colaboración con RTVE.

Fruto de los resultados satisfactorios del reto organizado en 2018 (Lleida-Solano et al., 2019a), se publicó en 2019 un expediente para el subtitulado automático de los informativos de los Centros Territoriales de RTVE contemplando, además, la particularidad de que todo el servicio se llevara a cabo en cloud. La Universidad Carlos III es la encargada de prestar esta facilidad a los Centros que realizan su informativo en castellano (Calero, 2020). En 2019 se realizó una nueva prueba de concepto (PoC) para el subtitulado de un informativo bilingüe, siendo capaz el sistema de detectar el cambio de idioma y acomodando los subtítulos al idioma hablado, con resultados positivos. En la actualidad se está trabajando para que las Comunidades con dos idiomas dispongan de este servicio.

En relación al análisis de grandes conjuntos de datos, RTVE ha desarrollado varias actividades de la mano de otras empresas españolas, junto a EFE y Narrativa (Corral, 2020) en los proyectos “Covid-19 Tracking Project" (2020) y “Data for hope" (2020), junto a Visiona en los estudios de igualdad de género promovidos por BBC (2020) y, actualmente, junto a Narrativa se están llevando a cabo pruebas para elaborar noticias sobre campeonatos de disciplinas deportivas que habitualmente no se cubren por falta de recursos y donde se dispone de datos digitalizados y estructurados.

Con el objetivo de poner en valor el Archivo del Fondo Documental RTVE se han llevado a cabo numerosas iniciativas que permitirán la integración futura de metadatos generados de forma automática en los actuales procesos de trabajo del archivo (Bazán-Gil; Guerrero-Gómez-Olmedo, 2018; Bazán-Gil et al., 2019; Bazán-Gil, 2020b). Fruto de estas experiencias, en diciembre de 2020, se publicó la convocatoria de licitación para el metadatado automático de contenidos del Fondo Documental RTVE, cuyo objetivo es establecer un servicio basado en inteligencia artificial en cloud para generar metadatos para 11.000 horas de video en un año (RTVE, 2020). 
En este escenario se ha desarrollado el proyecto para la segmentación automática de noticias para los informativos de $R N E$, que tiene como objetivos fundamentales mejorar la accesibilidad de estos programas mediante la transcripción automática del habla y el cortado de cada una de las noticias que lo componen para facilitar su consumo en distintas plataformas de publicación.

\section{Objetivo del proyecto}

En septiembre de 2019, el Departamento de Innovación Tecnológica de Radio Televisión Española (RTVE), con la colaboración de la Dirección de Medios de Radio Nacional de España (en adelante RNE), publicó un expediente de licitación para llevar a cabo una aplicación que, utilizando un sistema de automatización basado en inteligencia artificial, permitiera mejorar la experiencia de usuario de las personas que acceden a la web de RTVE, proporcionando noticias concretas de su interés, sin necesidad de escuchar todo el informativo.

La segmentación de programas radiofónicos constituye un reto tecnológico, mayor si cabe que la segmentación de programas de televisión, puesto que las noticias se encabalgan de forma muy rápida para no perder la atención del oyente y no se cuenta con el apoyo de la imagen como elemento de referencia adicional para el cortado de noticias.

La aplicación ofertada debía utilizar tecnologías del habla y procesamiento del lenguaje natural (PLN) para generar, de manera automática, la transcripción de voz a texto del programa como base para el posterior cortado y clasificación de las noticias. De esta forma no solo es posible ofrecer información personalizada para cada usuario, si no que se contribuye a mejorar la accesibilidad de los contenidos disponibles en la web de RTVE.

El expediente, publicado el 6 de septiembre de 2019, fue adjudicado a la empresa Aicox, quien a su vez integraba la tecnología de Etiqmedia (2020). Esta última es una empresa tecnológica española centrada en la accesibilidad (transcripción, subtitulado, etc.), la gestión de contenidos audiovisuales y la medición de la repercusión de protagonistas y marcas en radio, televisión y redes sociales. Sus soluciones se basan en tecnologías propias con un flujo de trabajo en tres fases: automatización, supervisión y explotación. Etiqmedia incorpora algoritmos de inteligencia artificial e interfaces que permiten la corrección de los errores y la explotación de los metadatos o su integración en gestores de contenidos multimedia. Una de las principales características de Etiqmedia es su capacidad para elaborar modelos propios para cada institución, utilizando sus datos y la retroalimentación proporcionada por los propios usuarios.

El programa seleccionado para este proyecto fue el informativo "Crónica de Madrid" (RNE, 2020a) que, con una duración de 50 minutos, se emite todos los días laborables a las 13:10 horas en la banda de FM, en la frecuencia de RNE (Radio 1). El programa se graba durante su emisión para su publicación posterior en la web de RTVE.

La elección de "Crónica de Madrid" se fundamentó en dos aspectos importantes: su estructura y el alcance de la información que ofrece. La estructura de este programa está claramente delimitada en bloques de información de actualidad, deporte, cultura y un bloque final que incluye una entrevista con algún protagonista de la jornada. Mientras el bloque informativo responde a una narrativa en las que se repite la fórmula locutor-noticia-locutor, el bloque que contiene la entrevista alterna preguntas y respuestas. En el primero, la locución responde a un guion, mientras en el segundo se produce un mayor protagonismo del habla espontánea. Todos estos factores garantizaban un modelo de lenguaje más amplio y, por tanto, extrapolable a otros programas radiofónicos de corte informativo. Por otra parte, el hecho de tratarse de información territorial limita, en líneas generales, el número de nombres de personas y entidades mencionadas, lo que facilita el estudio de la capacidad de aprendizaje de los actuales sistemas basados en machine learning.

En los requerimientos técnicos publicados en el expediente se solicitaba que el adjudicatario capturara la señal del informativo de la emisión en FM, la procesara obteniendo la transcripción a texto del audio, la segmentara por noticias, manteniendo la referencia temporal respecto del directo en el que ocurre, y clasificara las noticias según su temática. Los resultados debían estar disponibles en una plataforma web, con acceso restringido, controlado por usuario y contraseña, en un tiempo inferior a 15 minutos, a contar desde la finalización del programa. Toda la infraestructura necesaria para el procesamiento tenía que estar alojada en una nube, pública o privada, ajena a RTVE.

Así mismo, se solicitaba el uso de un modelo de lenguaje específico de actualidad informativa, adaptado a los informativos de $R N E$, que debía actualizarse periódicamente para incluir los nuevos términos que fueran surgiendo.

Para la clasificación de noticias se estableció como referencia IPTC Media Topics (IPTC, 2020) una taxonomía que se emplea para el intercambio de información en las agencias de noticias, medios digitales y prensa. Se actualiza periódicamente y está disponible en varios idiomas, entre ellos el español. Para facilitar el descubrimiento y mejorar la accesibilidad de los contenidos, cada tema identificado debía ilustrarse con un icono representativo.

La transcripción, segmentación y clasificación de los noticiarios debía realizarse exclusivamente a partir del propio contenido. Se estableció que no se facilitaría a la empresa adjudicataria escaletas o guiones ni tampoco material metadatado 
procedente del archivo de radio para el entrenamiento de su sistema, si bien podría ayudarse, como referencia para la segmentación, de elementos presentes en el audio como sintonías, cortinillas o "frases tipo" utilizadas frecuentemente por los presentadores para introducir una noticia o dar paso a la siguiente.

El proyecto se planificó en dos fases, una primera de cuatro meses para la puesta en marcha, configuración y entrenamiento del sistema, y una segunda, de prestación del servicio propiamente dicho, de 12 meses de duración. El paso de la primera a la segunda fase estaba condicionado a la superación de ciertos niveles de calidad que se fijaron en el $80 \%$ de aciertos para la transcripción del habla a texto y el $85 \%$ para la segmentación de noticias, si bien el paso a la segunda fase no implicaba en ningún caso que se paralizaran las mejoras en el sistema.

\section{Metodología del proyecto}

\subsection{Cronología del proyecto}

El expediente fue adjudicado a la empresa Aicox en noviembre de 2019, estableciéndose el 31 de marzo como fecha de finalización del periodo de configuración. A principios de diciembre, la interfaz para la validación de resultados estaba totalmente configurada y accesible para las personas responsables del control de proyecto. A partir de este momento comenzaron a procesarse los primeros programas y se establecieron reuniones periódicas con los adjudicatarios para estudiar los fallos encontrados y establecer los posibles puntos de mejora, que se iban recogiendo en las siguientes versiones de la herramienta. Las actualizaciones se han seguido realizando después de terminado el periodo de configuración, tal y como estaba previsto. Este proyecto, desde la redacción del expediente hasta la puesta en marcha de servicio, ha reunido a un grupo interdisciplinar de profesionales de RTVE formado por ingenieros, informadores y documentalistas, que ha trabajado de forma coordinada en las distintas fases aportando, en cada momento, su criterio profesional.

A mediados de marzo, debido a la situación excepcional ocasionada por la aparición del Covid-19 y el estado de emergencia decretado, la programación de RNE empezó a sufrir alteraciones diarias que afectaron a la emisión de "Crónica de Madrid". En su lugar, y de forma habitual, se emitieron ruedas de prensa o comparecencias en función de la actualidad informativa. Este tipo de contenidos no permitían un correcto seguimiento del proyecto, por lo que se decidió continuar el mismo con el programa informativo "14 horas" (RNE, 2020b) un programa de actualidad de alcance nacional y de una hora de duración.

La estructura de ambos programas responde a la de un informativo tipo, en el que, tras el sumario de las noticias más relevantes del día, el locutor da paso a cada una de las piezas, organizadas por bloques. Estos bloques incluyen además de actualidad, información meteorológica, tráfico, deporte y cultura y espectáculos, si bien el orden de los dos últimos bloques varía de un programa a otro. Tanto "Crónica de Madrid" como "14 horas" incluyen una entrevista de entre 2 y 6 minutos de duración y se despiden con un tema musical vocal. Si, desde el punto de vista de la estructura, los programas no varían, sí existen algunas variaciones en el modelo acústico (pronunciación de los sonidos de un idioma), modelo léxico (cómo se representan fonéticamente las palabras) y el modelo de lenguaje, especialmente en la narración del locutor principal.

El hecho de que se produjera este cambio en la emisión permitió comprobar cómo afectaba al rendimiento de un sistema, previamente ajustado a un programa, cuando éste era sustituido por otro de similares características, en cuanto a su estructura periodística, pero con presentadores diferentes, informadores distintos, mayor número de nombres de personajes y de lugares, etc., al tratarse de un informativo que ofrece noticias nacionales e internacionales, en lugar de locales.

\subsection{Implantación del servicio en fase de configuración y ajuste}

El servicio de cortado de noticias se aloja en una estación de trabajo, con tarjeta capturadora de audio, a la que llega la señal de FM a través de un sintonizador que está, a su vez, conectado a una antena de FM. Esta estación, que dispone también de una conexión a internet de $600 \mathrm{Mbps}$ simétricos, cuenta con un temporizador programable que pone en marcha el sistema a la hora de comienzo del informativo. Al activarse, captura la señal de emisión en la frecuencia de $R N E$ y la convierte en tiempo real en una pista de audio digital que se graba en el equipo.

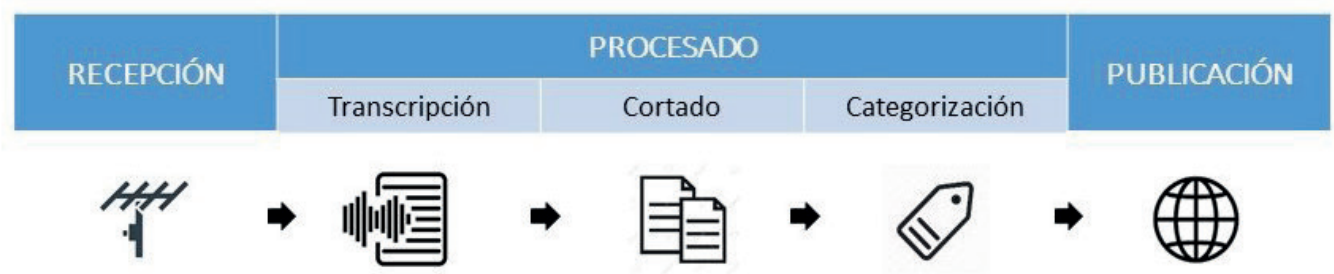

\begin{tabular}{|l|l|l|l|l|}
\hline Dispositivo & $\begin{array}{c}\text { Antena FM } \\
\text { Sintonizador }\end{array}$ & Estación de trabajo \\
\hline Funciones & $\begin{array}{l}\text { Recepción en } \\
\text { banda FM de } \\
\text { emisión RNE }\end{array}$ & $\begin{array}{c}\text { Transcripción a } \\
\text { texto de la locución }\end{array}$ & $\begin{array}{c}\text { Corte automático } \\
\text { de noticias }\end{array}$ & $\begin{array}{c}\text { Etiquetado en base } \\
\text { a ontología IPTC }\end{array}$ \\
\hline
\end{tabular}

Figura 1. Diagrama del sistema de cortado de noticias de $R N E$ 
Una vez concluido el informativo se realiza el procesado del audio en tres fases, cada una de las cuales corresponde a un módulo del sistema de Etiqmedia:

- Módulo de transcripción: En este módulo se realiza el proceso de transcripción del audio a texto. El sistema utiliza un reconocedor de voz automático, desarrollado por Etiqmedia, especializado en contenidos informativos, políticos e institucionales, que transcribe a texto la locución contenida en la pista de audio.

- Módulo de cortado: El segundo módulo se ocupa de la segmentación de cada una de las noticias que conforman el informativo. El sistema detecta, de forma automática, los diferentes hablantes que intervienen en el programa. Entre ellos localiza cuál es el hablante con mayor participación y lo establece como locutor principal o presentador, utilizando sus intervenciones como referencia para realizar cortes en el texto.

- Módulo de clasificación: En este módulo se lleva a cabo el proceso de clasificación de cada una de las noticias identificadas en la fase anterior. Mediante procesamiento del lenguaje natural (PLN), el sistema identifica palabras clave de las que es posible inferir un contexto temático y, en función de ello, asigna una categoría temática a cada noticia tomando como referencia la taxonomía Media Topics de IPTC. Por último, añade un recurso iconográfico que ayuda en la identificación visual.

Finalmente, el sistema sincroniza el fichero con el audio original del programa y el fichero JSON generado que contiene el resultado del proceso de transcripción, segmentación y clasificación. Ambos ficheros se envían a un portal web para su visualización. Este portal presenta una interfaz gráfica que facilita al usuario la localización de programas y la navegación por noticias e incluye un reproductor de audio, así como la generación automática de subtítulos en castellano para cada programa informativo.

En esta fase del proyecto la interfaz se utiliza para realizar el control de calidad y facilitar la retroalimentación para la mejora del sistema. Se trata por tanto de un entorno en pruebas cuyo grafismo deberá adecuarse a la imagen corporativa de RTVE una vez que se ponga en producción.

\subsection{Definición de los parámetros de calidad}

Durante la fase de configuración se definieron los criterios y métricas para la evaluación del sistema, reportando los problemas encontrados a Etiqmedia con el fin de mejorar su rendimiento. El análisis de la calidad se centró, en esta fase, en las funcionalidades consideradas críticas: transcripción de voz a texto y cortado de noticias, dejando la evaluación de la clasificación de noticias para fases posteriores, una vez que el sistema hubiera alcanzado niveles óptimos de rendimiento.

\subsection{Transcripción de voz a texto}

Para medir la calidad de la transcripción se empleó la tasa de error por palabra (WER en su acrónimo en inglés). EI WER permite establecer la fidelidad de una transcripción automática respecto a la transcripción real y se calcula teniendo en cuenta el número de inserciones, sustituciones y borrados respecto al número total de palabras. Aunque no hay una medida oficial de aceptabilidad, en base a los resultados del IberSpeech-RTVE Challenge 2018 (Lleida-Solano et al., 2019a), organizado por la cátedra RTVE-Universidad de Zaragoza (Lleida-Solano, 2020) en colaboración con la Red Española de Tecnologías del Habla, se considera que, para este tipo de programas, una transcripción automática aceptable no debería superar un $20 \%$ de errores.

Con estas premisas, se realizaron revisiones cualitativas de las transcripciones de los programas entre tres y cuatro veces por semana desde que la plataforma estuvo disponible, recogiendo los errores más comunes o repetidos y comunicándolos a los desarrolladores para ir mejorando el sistema.

A la finalización del periodo de configuración y entrenamiento, entre el 9 de marzo y el 6 de abril, se realizaron mediciones sistemáticas del WER durante cinco semanas de una muestra de 10 minutos de uno de los informativos de cada semana. Se eligieron franjas aleatorias, alternando entre el inicio, la mitad y el final del programa,

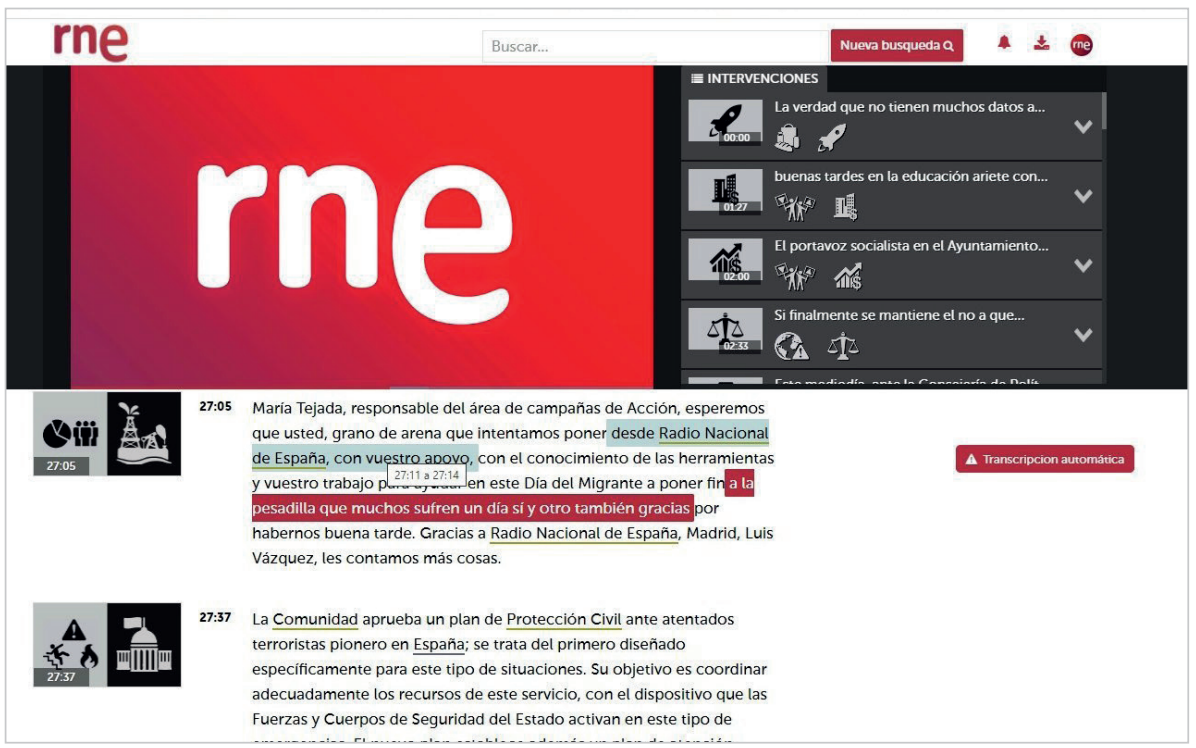

Figura 2. Imagen de la web de pruebas 
de forma que abarcaran los distintos bloques que tiene el informativo: resumen y titulares, entrevistas, noticias en general, deportes, cultura y despedida, etc. Cabe destacar que el cambio al informativo "14 horas" se produjo el día 24 de marzo, por lo que los dos últimos informativos analizados corresponden a este programa.

Adicionalmente, y para comprobar la validez de la tasa de error obtenida para estos segmentos, se calculó el WER para un informativo completo de 60 minutos. Para ello se utilizó Sclite incluida en el conjunto de aplicaciones SCTK (Speech Recognition Scoring Toolkit) del NIST (National Institute of Standards and Technology) y que se utilizan como herramienta de referencia en las competiciones Albayzin-RTVE Challenge 2018 (Lleida-Solano et al., 2019b) y 2020 (Lleida-Solano, 2020).

\subsection{Segmentación de noticias}

La calidad de la segmentación se basó en el cálculo de la exactitud entendida como la proporción entre los contenidos correctamente clasificados y el total de contenidos a clasificar. De acuerdo con la Dirección Técnica de RNE, se consideró que la exactitud no debía ser inferior al 85\% para la puesta en producción del portal.

La evaluación se hizo analizando un informativo cada semana, en días de la semana diferentes, desde enero hasta junio, excluyendo de la muestra a evaluar aquellos programas cuya emisión se vio afectada por cambios inesperados de programación. Para ello, dos evaluadores independientes comprobaron, para cada uno de los informativos analizados, la totalidad de los cortes generados por el sistema, si la noticia anterior era diferente a la siguiente y si el corte era preciso, es decir, si estaba hecho en el punto correcto o se había incluido palabras de una noticia en la otra. También se controló si había cortes que incluyeran más de una noticia diferente. Los aciertos se han medido en el primer caso en el porcentaje de noticias segmentadas correctamente frente al total de particiones realizadas y, en el segundo caso, en el porcentaje de noticias cortadas de forma precisa frente al total de noticias correctamente segmentadas.

\section{Resultados}

Analizamos, a continuación, los resultados de las pruebas llevadas a cabo. Se mantuvieron las fechas previstas para las pruebas a pesar del cambio al "14 horas", ya que no supuso un cambio en el modelo de lenguaje aplicado al sistema al tratarse, en ambos casos, de programas informativos muy parecidos en cuanto al vocabulario y estructura del lenguaje, además de que la mayoría de las noticias que se producen a nivel nacional están relacionadas con los términos incluidos en el modelo de Informativos de la Comunidad de Madrid.

\subsection{Calidad de la transcripción}

La tasa de error por palabras (WER) se calculó, como ya se ha mencionado, para fragmentos de 10 minutos y para un informativo completo. La tabla 1 muestra los resultados para cada fragmento analizado.

Tabla 1. Resultados de medidas de calidad en la transcripción

\begin{tabular}{|c|c|c|c|c|c|}
\hline \multirow[t]{2}{*}{ Informativo } & \multicolumn{3}{|c|}{ "Crónicas Madrid" } & \multicolumn{2}{|c|}{ "14 horas" } \\
\hline & (m1) & (m2) & (m3) & (m4) & (m5) \\
\hline \multicolumn{6}{|l|}{ Errores de transcripción } \\
\hline Sustituciones & 23 & 18 & 22 & 36 & 28 \\
\hline Borrados & 0 & 1 & 1 & 0 & 0 \\
\hline Inserciones & 37 & 7 & 13 & 42 & 32 \\
\hline Total palabras & 1.903 & 1.767 & 1.149 & 1.716 & 1.754 \\
\hline Total errores & 60 & 26 & 36 & 78 & 60 \\
\hline Tasa de error WER & $3,15 \%$ & $1,47 \%$ & $3,13 \%$ & $4,54 \%$ & $3,42 \%$ \\
\hline Aciertos en porcentaje & $96,85 \%$ & $98,53 \%$ & $96,87 \%$ & $95,46 \%$ & $96,58 \%$ \\
\hline
\end{tabular}

Las muestras 1 a 3 corresponden al programa "Crónica de Madrid", para los que se analizaron segmentos de 10 minutos al principio ( $\mathrm{m} 1$ y $\mathrm{m} 2$ ) y al final del informativo (m3). Para estas muestras las tasas de error se situaron entre el $1,47 \%$ (m2) y el 3,15\% (m1), no pudiéndose establecer una relación entre la franja de tiempo que ocupa la muestra analizada y la tasa de error. Las sustituciones y las inserciones son similares para todas las muestras, si bien el número de borrados es considerablemente mayor en la muestra 1. Las muestras 4 a 5 corresponden al programa " 14 horas" y los segmentos analizados corresponden a la penúltima y ultima franja del programa. Para estas muestras, la tasa de error varió en algo más de un punto y, si bien no se detectaron inserciones en ninguna de ellas, el número de sustituciones y borrados fue superior en la muestra 4. 
Traducido a porcentaje de aciertos, la media entre las 5 medidas fue del 96,8\%. Este resultado representa una muy buena transcripción y se justifica probablemente debido a las condiciones óptimas de entorno de la locución en un porcentaje de tiempo muy alto: grabación en estudio sin ruido ambiental, locutor profesional con buena dicción, velocidad adecuada de palabra y, también, por la tecnología de redes neuronales en la que se basa el sistema de transcripción.

La transcripción de un programa completo se realizó sobre la grabación del informativo del día 9 de marzo, el mismo al que corresponde la $\mathrm{m} 1$ de la evaluación anterior. La tasa de acierto obtenida para la transcripción completa fue del 96\%, lo que se supone una diferencia del 0,85\% respecto al resultado obtenido para el fragmento del programa.

Los errores de transcripción se concentraron fundamentalmente en los nombres propios, acrónimos, términos de nueva aparición y números. Veamos algunos ejemplos:

- Los nombres de locutores e informadores se transcribían de forma incorrecta en numerosas ocasiones: Así "Luz Cepeda" era transcrito como "Luis Cepeda"; "Ainhoa Caballero" como "y no a Caballero" o "Loreto Santos" como "Ios dos autos", por nombrar sólo algunos ejemplos.

- No se identificaban los acrónimos como ERTE, PCR o CIS como tales y, por tanto, su transcripción se realizaba en minúsculas. Este problema se corrigió en una nueva versión del sistema que se puso en producción en junio.

- No se identificaban términos de nueva aparición, ya fueran nombres propios como Covid, transcrito indistintamente como "cómic" o "covite", "Wuham" transcrito como "ucam", "Guajart" o "buzón" o comunes como "UCl" transcrito como "las luces" o "uncis" dependiendo del locutor.

- La transcripción de las cantidades y horas se realizaba de forma alternativa usando números o letras y, en ocasiones, una combinación de ambas formas como "veintidós 1.000 farmacias"; "veintitrés con dos por 100"; "Una de la tarde 27 minutos"; o "Ya son las dosis. Seis La uno seis en Canarias". Se realizaron modificaciones en el sistema que permitieron mejorar considerablemente la transcripción de cifras y horas pero que, sin embargo, afectaron a la transcripción del pronombre "uno" como reflejan frases del tipo "hermano de 1 de los"; " 1 de los puntos más".

La tabla 2 muestra una comparativa entre las frases originalmente pronunciadas y la transcripción generada.

En el caso de la puntuación y capitalización resulta complicado conseguir un método objetivo que permita una evaluación cuantitativa. El método más utilizado consiste en comparar los resultados del sistema con un texto patrón elaborado por un humano. Se realizó la prueba de entregar el mismo texto a dos evaluadores diferentes para su puntuación sin conseguir unanimidad respecto a la misma, por lo que se desestimó la realización de una evaluación de este tipo. De forma cualitativa se ha observado que, cuando el locutor está leyendo un texto previamente redactado, es decir, un lenguaje estructurado, se producen muy pocos errores, mientras que, si improvisa o si interviene un informador desde la calle en condiciones acústicas no óptimas, utilizando además un lenguaje natural, los errores en la transcripción aumentan de forma considerable.

\subsection{Calidad en la segmentación}

En lo que a la segmentación del informativo se refiere, se identificaron tres posibles comportamientos: la noticia se cortaba correctamente, el cortado incluía dos o más noticias o el cortado incluía parte de la noticia anterior o de la siguiente. Cuando la noticia se cortaba correctamente se trató de establecer además si el corte se realizaba en el punto preciso. El gráfico 1 muestra los resultados obtenidos.

Del número de muestras analizadas, 16 en total, las primeras ocho (m1-m8) corresponden a "Crónica de Madrid", mientras que las ocho siguientes (m9-m16) son de "14 horas". Se comprobó que el porcentaje de noticias bien segmentadas (cortes correctos), fluctuaba entre el $81,25 \%$ y el $96,15 \%$. De esas noticias correctamente segmentadas,

Tabla 2. Ejemplos de errores detectados en la transcripción

\begin{tabular}{|l|l|}
\hline Transcripción manual & Transcripción automática \\
\hline gracias Paco, una de la tarde & desgracias vacuna de la tarde \\
\hline a última hora & al Timma, hora \\
\hline Venir con cita previa & ver el concierto previo \\
\hline Bueno, está aquí ya & buena taquilla \\
\hline la van a & La Habana \\
\hline Nudo de Manoteras & nudo de loteras \\
\hline la BESCAM & la ves. Cam \\
\hline Se lo avanzábamos en titulares & eso lo bonzo en los titulares \\
\hline
\end{tabular}

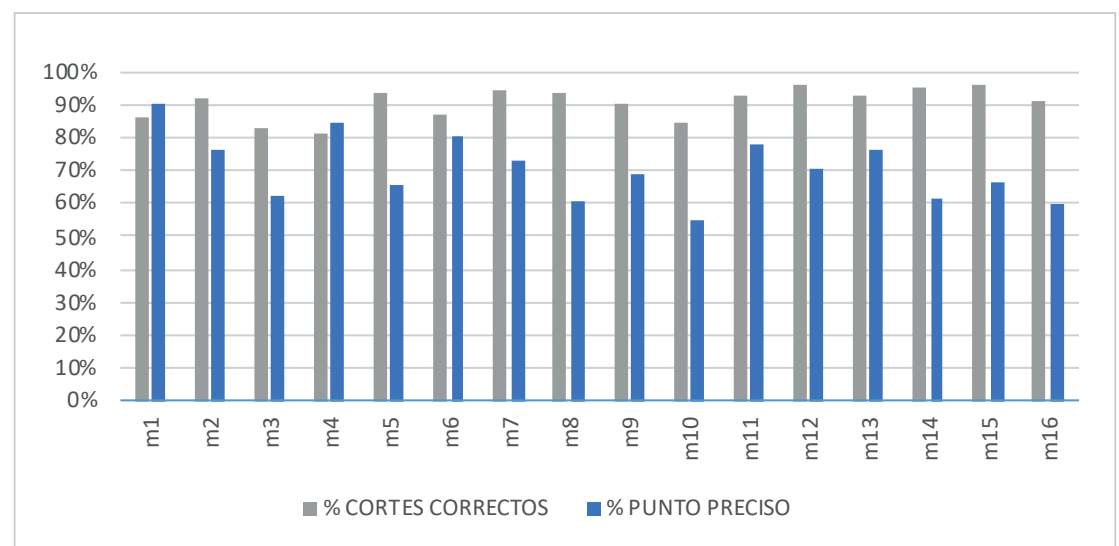

Gráfico 1. Resultados de medidas de calidad en la segmentación 
entre un $55,26 \%$ y un $90,68 \%$ lo están, además, en la palabra exacta donde cambia la noticia (punto preciso). El número de noticias extraídas por el sistema en ambos informativos es muy diferente, entre 30 y 37 en el caso de "Crónica de Madrid" y entre 41 y 55 en "14 horas". Sin embargo, no se ha podido establecer ninguna relación entre el número de cortes realizados y la exactitud de los mismos, ni su precisión. De la muestra analizada, once programas han superado el $90 \%$ en aciertos en la segmentación, la mayoría de los cuales, siete en concreto, corresponden a "14 horas". En el caso de la precisión en el corte, únicamente en "Crónica de Madrid" se supera el 80\% de aciertos.

Una de las variables que el sistema utiliza para considerar un cambio de noticia es la detección de cambio de hablantes, sin embargo, sucede frecuentemente que el sistema no logra distinguir el punto exacto en el que se produce este cambio y, por tanto, falla al separar las frases ortográficamente. Esto es así porque la ventana en la que se analiza si hay o no cambio de hablante es de algún segundo (en un segundo se pueden decir 3 ó 4 palabras), cuando debería ser de milisegundos para que la detección del cambio fuera precisa.

Un análisis más detallado de los resultados nos llevó a determinar que los cortes incorrectos estaban condicionados también por la ausencia de pausas en la locución principal y, como consecuencia de ello, las frases no se puntuaban correctamente, produciéndose además errores en la identificación de los hablantes. En términos generales, el sistema presentaba dificultades en alguna de estas circunstancias:

- Se producía un intercambio de saludos entre el locutor principal y el periodista que narra la noticia o cuando la noticia comienza con el saludo del segundo.

- En el programa "Crónica de Madrid", el locutor principal usaba fórmulas de despedida que incluían la palabra gracias y el nombre del redactor. El sistema no hacía una interpretación correcta, dejando "gracias" como final de una noticia y el nombre del redactor en el inicio de la siguiente. En función de un primer análisis y para "Crónica de Madrid" se estableció una regla de cortado por la cual el sistema establecía un cambio de noticia detrás de esta fórmula, lo que contribuyó a mejorar considerablemente la tasa de acierto en el cortado y, como consecuencia de ello, la pertinencia en la clasificación de las noticias. Sin embargo, esta regla no resultó útil al cambiar al informativo "14 horas", en el que la frase de despedida del locutor no respondía a la fórmula anterior, sino que utilizaba "gracias" seguida del nombre del informador añadiendo a continuación "buenas tardes".

- El locutor introducía la siguiente noticia sin despedir al periodista e incluía, una vez iniciada la narración, la formula "gracias" seguida del nombre del informador para continuar después con su discurso.

- Se producía un encabalgado de noticias, es decir, el locutor principal narraba dos o más noticias sin intervención de otro hablante. La siguiente frase es un ejemplo de este error de cortado: "Mantenemos línea abierta con Palacio Cibeles, allí volvemos en unos instantes, la investidura de Pedro Sánchez, cambiemos de argumento, sigue alimentando..."

- No tenía en cuenta las cortinillas musicales que anuncian el cambio de sección dentro del programa.

\section{Conclusiones}

El servicio de cortado de noticias ha obtenido un rendimiento adecuado. Tras el periodo de configuración y ajuste se han alcanzado los niveles de calidad establecidos tanto para la transcripción automática como para la segmentación de noticias.

En términos generales, y respecto a la transcripción automática, se puede afirmar que el servicio se ha mantenido en tasas de acierto superiores al $90 \%$, siendo menor la incidencia de errores en los segmentos del programa en los que la locución predominante responde a un guion, es pausada y está correctamente entonada frente a los segmentos en los que predomina el habla espontánea o se superponen los hablantes. Se ha detectado un mejor rendimiento del sistema cuando la locución se realiza en estudio frente a las conexiones telefónicas con ruido ambiente y una menor calidad de señal de audio. Así mismo, durante el periodo de configuración, se estableció que el sistema tenía problemas para detectar y, por tanto, para transcribir términos de nueva aparición como Covid, por lo que junto al equipo de Etiqmedia se trabajará en mejorar los tiempos de respuesta del sistema ante los nuevos términos. Para ello se trabajarán técnicas que permitan multiplicar los textos que las contienen, sometiendo al sistema a técnicas de sobrentrenamiento.

En lo que a la segmentación se refiere, es interesante señalar que esta es la primera aproximación al cortado automático de noticias que se realiza en RTVE en el ámbito radiofónico y, como tal, la precisión se ha considerado adecuada, si bien, se asume que es precisamente aquí donde la automatización tiene una mayor capacidad de mejora. En este sentido, se ha previsto que el sistema use como referencia las cortinillas y sintonías para un cortado más preciso de las noticias y que la transcripción se inicie, precisamente, a partir de éstas y no a partir de las señales horarias, ya que no siempre coinciden con el principio y el final de cada programa.

Por otro lado, se va a trabajar en mejorar la capacidad del sistema para identificar y segmentar la locución por hablantes. Actualmente, aunque la automatización detecta que hay un cambio de hablante, no lo hace con la precisión adecuada. Establecer las fronteras entre hablantes es un pilar básico del sistema. Mejorar esta capacidad no solo redundará en 
una mejor segmentación de las noticias sino también en la mejora en la puntuación y capitalización, lo que hace que la transcripción sea más compresible. En esta línea se espera mejorar la representación gráfica de la noticia diferenciando a los hablantes por colores y estableciendo saltos de párrafo. Adicionalmente se trabajará en la clasificación temática de las noticias y en los iconos representativos que las acompañan.
Los resultados ponen de manifiesto que, a pesar de ser tecnologías razonablemente maduras, son necesarios procesos de ajuste y aprendizaje en el que la intervención humana sigue teniendo un papel relevante

El cambio de programa objeto del servicio no supuso una disminución apreciable de la calidad del mismo. Esto supone que, con independencia de la cabecera, el sistema es adecuado para los programas de corte informativo con una estructura similar. Por otra parte, se ha constatado que la flexibilidad del sistema para adaptarse a las particularidades de un programa es relevante para mejorar las tasas de acierto, especialmente en el cortado de noticias, y, sin embargo, una excesiva adecuación a estas particularidades puede ser contraproducente cuando se cambia de programa. En el futuro se realizarán pruebas para analizar el comportamiento del sistema en programas no informativos.

En resumen, este proyecto ha puesto de manifiesto que la aplicación de soluciones de inteligencia artificial en $R T V E$ ofrece numerosas oportunidades desde el punto de vista de la difusión de los contenidos de radio y televisión, ya que permite una mayor adaptación de éstos a los requisitos de publicación en medios de comunicación social y plataformas de consumo y, al mismo tiempo, permite presentar una oferta más personalizada y accesible para los usuarios.

\section{Bibliografía}

Associated Press (2020). Automated insigts customer stories. https://automatedinsights.com/customer-stories/associated-press

Bazán-Gil, Virginia (2018). “El renacimiento de los archivos: inteligencia artificial y semántica aplicada a la descripción de contenidos audiovisuales". En: IX Encuentros de Centros de Documentación de Arte Contemporáneo. Explotación, Integración y Difusión de Conocimiento de las Instituciones Patrimoniales.

https://es.slideshare.net/Artium_Vitoria/ix-encuentros-de-centros-de-documentacin-de-arte-contemporneo-en-artiumvirginia-bazn-gil

https://biblioteca.artium.eus/Record/164617

Bazán-Gil, Virginia (2020). "Artificial intelligence: an object of desire". In: FIAT/IFTA, 13 May. http://fiatifta.org/index.php/media/archivalreads/archivalreads-artificial-intelligence

Bazán-Gil, Virginia; Guerrero-Gómez-Olmedo, Ricardo (2018). “Descripción automática de archivos audiovisuales: NeuralTalk, un modelo de video2text aplicado al archivo de RTVE". BiD: Textos universitaris de biblioteconomia i documentació, n. 41. https://doi.org/10.1344/BiD2018.41.7

Bazán-Gil, Virginia; Lleida-Solano, Eduardo; Pérez-Cernuda, Carmen; Gómez-Zotano, Manuel J.; De-Prada, Alberto (2019). "Tecnologías del habla: nuevas oportunidades para los archivos de televisión". En: 14o Congreso ISKO España. http://eprints.rclis.org/38447/1/CatedraRTVEUnizar_ISKO2019.pdf

$B B C$ (2020). The equality project 50:50.

https://www.bbc.co.uk/5050

Calero, Juan F. (2020). "La inteligencia artificial que lleva a otro nivel el subtitulado automático en informativos de TV o conferencias". Madritd, 23 julio.

http://www.madrimasd.org/notiweb/noticias/inteligencia-artificial-que-lleva-otro-nivel-subtitulado-automatico-eninformativos-tv-o-conferencias

Cátedra RTVE (2017).

http://catedrartve.unizar.es

Corral, David (2020). "Periodismo tecnológico o ¿tecnología para el periodismo? En tiempos de pandemia". RTVE, 30 abril. https://www.rtve.es/rtve/20200430/periodismo-tecnologico-tecnologia-para-periodismo-tiempos-pandemia/2013145.shtml

Data for hope (2020).

https://dataforhope.com

Doukhan, David (2019). "Gender equality monitor". In: FIAT/IFTA World Conference in Dubrovnik. https://es.slideshare.net/fiatifta/doukhan-gender-equality-monitor

EBU (2019). The next newsroom: Unlocking the power of Al for public service journalism. News report 2019.

https://www.ebu.ch/publications/strategic/login_only/report/news-report-2019

Etiqmedia (2020). Etiqmedia. Radio indexing.

http://www.etiqmedia.com/soluciones/radio-indexing.php 
Fraunhofer-Gesellschaft (2019). "Software that can automatically detect fake news". Phys org, February 1. https://phys.org/news/2019-02-software-automatically-fake-news.html

Galvez, Giovanni (2020). "Al machine translation of subtitling for live news and sports". In: MDN Workshop. https://tech.ebu.ch/contents/publications/presentations/2020/mdn2020/ai-machine-translation-of-subtitling-for-livenews-and-sports.html

Graves, Lucas (2018). Understanding the promise and limits of automated fact-checking. Reuters Institute. https://reutersinstitute.politics.ox.ac.uk/sites/default/files/2018-02/graves_factsheet_180226\%20FINAL.pdf

Grothaus, Michael (2019). "Machine learning isn't effective at identifying fake news". Fast Company, 15 October. https://www.fastcompany.com/90417625/machine-learning-isnt-effective-at-identifying-fake-news

Herrero-Diz, Paula; Varona-Aramburu, David (2018). “Uso de chatbots para automatizar la información en los medios españoles". El profesional de la información, v. 27, n. 4, pp. 742-749.

https://doi.org/10.3145/epi.2018.jul.03

IPTC (2020). News codes.

https://iptc.org/standards/newscodes

Jones, Bronwyn; Jones, Rhianne (2019). "Public service chatbots: Automating conversation with BBC News". Digital journalism, v. 7, n. 8, pp. 1032-1053.

https://doi.org/10.1080/21670811.2019.1609371

Lempinen, Jaakko; Kokko, Jan; Matusiak, Marek (2020). “Applications of automated media extraction from Yle Areena videos". In: MDN Workshop.

https://tech.ebu.ch/contents/publications/presentations/2020/mdn2020/applications-of-automated-media-extractionfrom-yle-areena-videos.html

León-Carpio, Antonio; López-De-Quintana, Eugenio (2020). “Artificial Intelligence for a role change in television archives: the Atresmedia experience". In: IASA - FIAT/IFTA.

https://2020iasafiatiftaconference.sched.com/event/eLpP

Lleida-Solano, Eduardo (2020). Iberspeech 2020 evaluation challenges. Cátedra RTVE - Universidad de Zaragoza, Albayzín Evaluations.

http://catedrartve.unizar.es/albayzin2020.html

Lleida-Solano, Eduardo; Ortega-Giménez, Alfonso; Miguel, Antonio; Bazán-Gil, Virginia; Pérez-Cernuda, Carmen; Gómez-Zotano, Manuel; De-Prada, Alberto (2018). RTVE2018 Database Description.

http://catedrartve.unizar.es/reto2018/RTVE2018DB.pdf

Lleida-Solano, Eduardo; Ortega-Giménez, Alfonso; Miguel, Antonio; Bazán-Gil, Virginia; Pérez-Cernuda, Carmen; Gómez-Zotano, Manuel; De-Prada, Alberto (2019a). "The IberSpeech-RTVE challenge on speech technologies for Spanish broadcast media". Applied sciences, v. 9.

https://www.mdpi.com/2076-3417/9/24/5412

Lleida-Solano, Eduardo; Ortega-Giménez, Alfonso; Miguel, Antonio; Bazán-Gil, Virginia; Pérez-Cernuda, Carmen; GómezZotano, Manuel; De-Prada, Alberto (2019b). “Albayzin 2018 evaluation: The IberSpeech-RTVE”. Applied sciences, n. 22. https://www.mdpi.com/2076-3417/9/24/5412/pdf

Lleida-Solano, Eduardo; Ortega-Giménez, Alfonso; Miguel, Antonio; Bazán-Gil, Virginia; Pérez-Cernuda, Carmen; Gómez-Zotano, Manuel; De-Prada, Alberto (2020). RTVE2020 Database Description.

http://catedrartve.unizar.es/reto2020/RTVE2020DB.pdf

LSE (2020). JournalismAI Case studies. The London School of Economics and Political science. https://www.Ise.ac.uk/media-and-communications/polis/JournalismAl/Case-studies

Molumby, Conor; Whitwell, Joe (2019). “General election 2019: Semi-automation makes it a night of 689 stories". BBC news labs, 13 diciembre.

https://bbcnews/abs.co.uk/news/2019/salco-ge

Nixon, Lyndon (2020). “Metadata-driven TV content repurposing and republication”. In: MDN Workshop. https://tech.ebu.ch/contents/publications/events/presentations/mdn2020/metadata-driven-tv-content-repurposingand-republication

Opoku-Boateng, Judith; Asano, Jun (2020). "NHK's diversification of search methods using Al". In: IASA - FIAT/IFTA conference. https://2020iasafiatiftaconference.sched.com/event/eLoy

Parmentier, Matthieu (2020). "Analysing political debates to feed data journalists". In: MDN Workshop.

https://tech.ebu.ch/contents/publications/presentations/2020/mdn2020/analysing-political-debates-to-feed-datajournalists.html 
Prensa RTVE (2018). RTVE lanza el proyecto 'Journalism Innovation Hub' para estudiar la transformación digital de los informativos.

https://www.rtve.es/rtve/20180222/rtve-lanza-proyecto-journalism-innovation-hub-para-estudiar-transformacion-digitalinformativos/1682664.shtml

Proyecto covid 19 (2020).

https://covid19tracking.narrativa.com

Rath, Sid; Veerwaijen, Christiaan; Forster, Christoph (2020). "Al-enabled hyper-tagging engines for football archives". In: IASA - FIAT/IFTA Conference.

https://2020iasafiatiftaconference.sched.com/event/eLoj

Rehm, Georg (2020). Research for CULT Committee - The use of artificial intelligence in the audiovisual sector. European Parliament, Policy Department for Structural and Cohesion Policies, Brussels.

https://www.europarl.europa.eu/thinktank/en/document.htm/?reference=IPOL_IDA(2020)629221

$\operatorname{ReTV}(2020)$.

https://retv-project.eu

RNE (2020a). Madrid Informativos de RNE.

https://www.rtve.es/alacarta/audios/informativo-de-madrid

RNE (2020b). RNE 14h.

https://www.rtve.es/alacarta/audios/14-horas

Rozalén-Serrano, Miguel-Ángel; Aranda-Jiménez, Álvaro (2020). “Generación automática de palabras clave para monitorizar dominios en redes sociales”. En: Actas del IV Congreso ISKO España-Portugal 2019, XIV Congreso ISKO España 2019. https://dialnet.unirioja.es/servlet/libro?codigo $=766025$

Rozalén-Serrano, Miguel-Ángel; Aranda-Jiménez, Álvaro; Rodríguez, Francisco; Álvarez-Rodríguez, José-María (2020). Proyecto Social Media Radar. Madrid. ISBN: $108283064 X$

RTVE (2019). Portal de licitaciones.

https://licitaciones.rtve.es/licitacion/licitaciones/detalle?id=744264

RTVE (2020). Portal de licitaciones.

https://licitaciones.rtve.es/licitacion/licitaciones/detalle?id=1208797

Saarikoski, Lauri (2020). "How do the end-users find all this automated metadata?". In: MDN Workshop.

https://tech.ebu.ch/contents/publications/presentations/2020/mdn2020/how-do-the-end-users-find-all-this-automatedmetadata.html

Sanjinés, Diana (2020a). "Cómo RTVE implementa la personalización de contenido en sus aplicaciones". Noticias OI2. http://oi2media.es/2020/07/03/como-rtve-implementa-la-personalizacion-de-contenido-en-sus-aplicaciones

Sanjinés, Diana (2020b). "OI2 publica el tercer informe sobre periodismo e inteligencia artificial". Publicaciones OI2. http://oi2media.es/2020/11/06/oi2-publica-el-tercer-informe-sobre-periodismo-e-inteligencia-artificial

Túñez-López, Miguel; Toural-Bran, Carlos; Cacheiro-Requeijo, Santiago (2018). “Uso de bots y algoritmos para automatizar la redacción de noticias: percepción y actitudes de los periodistas en España". El profesional de la información, v. 27, n. 4, pp. 750-758.

https://doi.org/10.3145/epi.2018.jul.04

Túñez-López, Miguel; Toural-Bran, Carlos; Valdiviezo-Abad, Cesibel (2019). “Automatización, bots y algoritmos en la redacción de noticias. Impacto y calidad del periodismo artificial". Revista latina de comunicación social, n. 74, pp. 1411-1433. https://doi.org/10.4185/RLCS-2019-1391

Vállez, Mari; Codina, Lluís (2018). "Periodismo computacional: evolución, casos y herramientas". El profesional de la información, n. 27, n. 4.

https://doi.org/10.3145/epi.2018.jul.05

Van-Rijsselbergen, Dieter (2020). "Metadata proccessing in the H2020 Memad prototype platform”. In: MDN Workshop. https://tech.ebu.ch/contents/publications/presentations/2020/mdn2020/metadata-processing-in-the-h2020-memadprototype-platform.html

WashPostPR (2017). "The Washington Post leverages automated storytelling to cover high school football”. Washington Post, 1 September.

https://www.washingtonpost.com/pr/wp/2017/09/01/the-washington-post-leverages-heliograf-to-cover-high-school-football

Yle (2018). Yle releases code for 'robot journalist' Voitto.

https://yle.fi/uutiset/osasto/news/yle_releases_code_for_robot_journalist_voitto/10126261 